diffusers having a large number of orifices and a low rate of flow through each are in some cases smaller than would be predicted from the theoretical equations. A mechanism to account for this has been suggested and confirmed experimentally by Pattle. The properties of the liquid have a pronounced effect in these cases. If a mycelium is built up during fermentation, bubbles become trapped and coalesce. In Mr. Bowers's experiments the rate of absorption of oxygen from bubbles formed with a porous ceramic plate was reduced by a factor of 20 by 2 per cent of paper pulp added to simulate mycelium. This factor was reduced to approximately 7 by agitating the liquid above the plate with a turbine-type stirrer. In an apparatus in which dispersion of the gas was brought about by the mechanical agitator itself, the effect of the paper pulp was reduced still further to a factor of about 2 .

Mr. P. E. Sawyer presented a paper by Prof. F. H. Garner and himself on surface-active effects within gas bubbles. The presence of surface-active agents in solution may appreciably influence the behaviour of rising gas bubbles owing to the formation of an adsorbed film at the bubble interface. The principal effect of such an adsorbed interfacial film is to modify the hydrodynamic properties at the gasliquid interface. Normally the interface between a rising bubble and a liquid permits the transmission of viscous shear across it, resulting in the formation of toroidal circulation currents within the bubble. The drag exerted by the liquid on a circulating bubble is lower than that exerted if the bubble acts as a solid spheroid, and the velocity of ascent of the bubble is thus increased. The presence of a surfaceactive agent in the liquid may produce a semi-rigid or rigid structure at the interface which resists the transmission of shear and may inhibit or completely suppress circulation.

Mass transfer between a rising bubble and a liquid is also dependent on the character of the interfacial film. Internal circulation is accompanied by a considerable reduction in the resistance to mass transfer in both phases. Thus an adsorbed film will reduce mass transfer-rates by inhibiting circulation; in addition, the film may itself constitute a resistance to mass transfer, possibly by molecular interaction or by mechanical obstruction. The presence of surfaceactive agents may have an important secondary effect upon the path and shape of a rising bubble. Over a certain velocity-range bubbles normally rise with a spiral motion; surface contamination, however, changes this into a zig-zag motion in one plane. The reduction in surface tension produced by surfaceactive agents may also lead to changes in the shape of the bubble and to an increased tendency to oscillation.

The effects of certain variable factors on the formation and behaviour of gas bubbles in water, some of which had been referred to by the speakers, were strikingly demonstrated, in slow motion, in a short film. The effects of varying the rate of flow of gas, the diameter of the hole from which the bubbles were formed, and the motion of the water were shown. Dyes injected into the liquid or polarized light were used for illustrating the characteristics of the fluid dynamics around the bubble.

Dr. G. N. Rolinson dealt with the effects of aeration on fungal metabolism. One of the striking differences between moulds and bacteria is that there are no anæerobic moulds. It is extremely doubtful whether any growth takes place when fungi are completely deprived of oxygen. Aeration is frequently a limiting factor in fungal cultures, increased aeration increasing both the amount and rate of growth, and increased aeration frequently results in an increased rate of respiration, since a more extensive respiratory mechanism is synthesized. The respiratory quotient of mycelium grown under conditions of high aeration may be more than double that of mycelium grown under low aeration. One respiratory mechanism developed in response to the level of aeration appears to be the cytochrome system. Mycelium of Penicillium chrysogenum oxidizes lard oil rapidly when grown under conditions of high aeration, but only slowly when grown under conditions of low aeration. High rates of aeration are essential for high yields of penicillin. Maximum rate of penicillin production by washed mycelium of $P$. chrysogenum has been found to be dependent on the mycelium being permitted to respire at its maximum rate. This is similar to effects observed in citric acid production whereas, in contrast, the maximum production of $\alpha$-amylase occurred when the rate of respiration was below the maximum. In addition to the yeasts, many of the Mucors and Fusaria also carry out vigorous alcoholic fermentation when deprived of air. Under aerobic conditions, alcoholic fermentation is depressed, and so also is the rate of glycolysis. This, the Pasteur effect, is probably caused by a lack of inorganic phosphate under aerobic conditions. Aeration may also effect metabolism by sweeping away carbon dioxide which might otherwise be assimilated.

The subjects raised in the lively discussion following the papers covered a wide field. Questioners were interested in methods of deoxygenation of water, in the design of absorption vessels and stirring mechanisms and in the performance of different types of aeration systems. Methods of measurement of dissolved oxygen concentrations were considered. Several speakers cited instances in which polarographic techniques had been used with success. The general opinion was that aeration studies could be of considerable value in improving practical fermentation processes, although rate of aeration was not always a factor limiting rate of production. Impedances in the organisms themselves were sometimes the controlling factor. In replying to questions arising out of his paper, Dr. Rolinson said that yeasts grown aerobically would probably not grow afterwards in complete absence of oxygen, although some growth might occur in partially anaerobic conditions. With regard to the Pasteur effect, this is not a permanent alteration of the enzyme system. He agreed that impedance across the cell wall cannot readily be distinguished from inability of enzyme systems to utilize oxygen when it became available.

\section{TREND AND PATTERN OF FERTILITY IN GREAT BRITAIN}

7 HE sixth, and final, volume of the Papers of the Royal Commission on Population describes the family census of 1946-its origins, methods and defects-and goes on to analyse in great detail the information collected. The joint authors are Prof. D. V. Glass, of the London School of Economics, and Prof. E. Grebenik, of the University of Leeds.

The family census was taken because some of the most important demographic questions with which the Royal Commission on Population was concerned 
could not be answered in any other way. Since the normal census machinery could not be used, the family census was trken on a sample basis, the desired information being collected from a 10 per cent random sample of the women in Great Britain who were, or had been, married. It had no statutory sanction, but just over 87 per cent of the women responded-a very high response for a voluntary investigation. The extent of bias arising from nonresponse was examined, and it was found that any effect on the validity of the results could be safely discounted.

In part, the examination of the results follows the pattern customarily found in fertility censuses, in which the basic tables relate to the total number of children which the women had had by the time of the census. These tables show, for example, that of the women covered by the family census, 66 per cent had had not more than two children, and only 9 per cent had had six or more. On average, each woman had $2 \cdot 3$ live births by the time of the census: dividing the women into two broad social groups, manual and non-manual (based on the husband's occupation), the corresponding averages are 2.52 and 1.90 live births.

Study of the information by date of marriage shows clearly the decline in family size : for example, a woman married in 1920 had only about half as many children as a woman married before 1890 about $2 \cdot 5$ children in the former case, and about 5 in the latter. Whereas in the late nineteenth century families of five or more children constituted threefifths of all families, the marriages of 1925 were dominated by one- and two-child families. Over this period, too, childlessness had doubled in frequency.

The statistics for all marriages (including childless marriages) show a consistent fall in family size with increasing age at marriage. But it is also shown that the relation between age at marriage and final family size is becoming somewhat less rigid. This is the kind of development which would be expected with the increasing acceptance of 'family planning' and with the spread of the small family.

A special feature of the family census was that the date of birth of each child was recorded. This information made possible the comparison of the families of different groups of marriages after different durations of marriage. For example, it was possible to compare the families, after six years of marriage, of women married in 1930 with the families, after six years of marriage, of those married in 1940 . Such comparisons have enabled the authors to give a much clearer picture of current trends, and to make some tentative suggestions about the future.

The statistics show that the fall in family size has been accompanied not so much by a more widespread spacing between births, as by a contraction in the spen of years during which children are born.

The general trend of ultimate family sizes, as deduced from the partial records available, shows a continued but slow fall in size of completed family for the marriages up to 1930 , a fluctuating stability for the marriages of 1931-40, and thereafter $a$ suggested increase in family size.

The data on family sizes have also been used for constructing replacement-rates indicating whether, with a continuance of fertility, marriage and mortality at certain specified levels, the population would ultimately increase or fall. For the women born in 1895-99, the estimated rate is only $0 \cdot 74$, well below the 1.0 which would show adequate long-term replacement of population. For more recent generations the replacement-rates will be higher; but the complete record of childbearing is not yet available. Marriage-rates have, however, been calculated and give a reasonably clear picture of the situation. Taking the marriages of 1941-43, the estimated rate ranges between 0.94 and 0.98 , and this, although based in part on projected data because the families of these women are not completed, serves to suggest that the replacement-rates likely to result from recent marriages are not far from $1 \cdot 0$.

Far greater difficulties arise in attempting to predict the future of family size. Examination of the data suggests that family limitation is even more widespread in Britain than has been previously estimated, and that a further substantial fall in fertility is unlikely to occur simply through an extension of family planning. The analysis suggests that a new stability has developed in recent years, and that there may in fact have been some increase in family size since the Second World War. These developments have accompanied the spread throughout the community of the small-family pattern; nothing so far visible in the statistics foreshadows a major departure from that pattern in the near future.

\section{NATIONAL RESEARCH DEVELOPMENT CORPORATION}

\section{REPORT FOR 1953-54}

$\mathrm{T}$ HE chief feature of the report and statement of accounts of the National Research Development Corporation for the year ended June 30, 1954*, is the quinquennial review of the Corporation's work, and this will be discussed separately. Among the new development projects in which the Corporation has interested itself during the year is a stereoscopic ciné camera, the development of which might be advantageous for educational, medical, scientific and technological purposes, and a technique for simplifying and economizing in the wiring of electric circuits.

Of projects already referred to in previous reports, the electronic digital computer continues to be the Corporation's largest single project, more than $£ 325,000$ of the $£ 928,000$ borrowed by the Corporation representing stocks, work in progress or patent expenditure in this field. Six big computers of the University of Manchester type have been ordered and have been or are being built for the Corporation's account, of which five have been sold and will have been delivered during 1954-55. The much less costly 401 Mark I computer has been transferred to Rothamsted Experimental Station after completing its trials at the Mathematical Laboratory, Cambridge, and is in routine use for calculations relating to agricultural statistics and other purposes. The design of a further computer embodying many of the successful devices in the prototype 401 has also been decided. The Packman potato harvester is now out of the development stage, and a number of machines are being made for the harvest this year. The Ricardo light steam-engine is now available for exploitation, and good progress has been made with the process of extracting hecogenin from sisal juice

- National Research Development Corporation. Report and Statement of Accounts for the Year 1st July, 1953, to 30th June, 1954 Pp. 20. (London: H.M.S.O., 1955.) $9 d$. net. 